\title{
El QUEHACER DEL PROFESIONAL DE LA PSICOLOGÍA DE LA SALUD: DEFINICIONES Y OBJETIVOS
}

\author{
Laura Edith Pérez-Laborde y Liliana Moreyra-Jiménez \\ FES Iztacala, UNAM \\ México
}

\section{RESUMEN}

La psicología de la salud es un campo de aplicación que surgió como respuesta ante la necesidad de comprender los factores psicológicos involucrados en el proceso de salud-enfermedad con el objetivo de generar acciones efectivas de promoción de la salud y prevención de la enfermedad. Se han hecho múltiples propuestas para definirla, creado o adaptado diversos modelos teóricos y herramientas para responder a sus objetivos. Sin embargo, en la práctica se suele confundir con otras áreas de la psicología. El presente trabajo pretende clarificar el quehacer del profesional de la psicología de la salud respecto a los elementos fundamentales que la definen y constituyen, se revisan sus objetivos, los niveles de intervención preventiva, los campos de acción y las diferencias entre psicología de la salud y otras áreas. Todo ello con la intención de facilitar su comprensión a quienes se interesen en el estudio de esta área de aplicación.

\section{Palabras Clave:}

Psicología de la salud, promoción de la salud, prevención de la enfermedad, modelos en psicología de la salud, competencias en psicología de la salud.

\section{THE WORK OF THE HEALTH PSYCHOLOGY PROFESSIONAL: DEFINITIONS AND OBJECTIVES}

\begin{abstract}
Health psychology is a field of application that emerged as a response to the need to understand the psychological factors involved in the disease health process in order to generate effective actions for health promotion and disease prevention. There have been multiple proposals to define it and created or adapted various theoretical models and tools to respond to its objectives. However, in practice it is often confused with other areas of psychology. This paper aims to clarify the work of the health psychology professional with respect to the fundamental elements that define and constitute it, review its objectives, the levels of preventive intervention, the fields of action and the differences between health psychology and other areas. All this with the intention of facilitating their understanding to those who are interested in the study in this area of application.
\end{abstract}

Keywords:

Health psychology, health promotion, disease prevention, models in health psychology, competencies in health psychology.

Bitácora del Artículo:

| Recibido: 1 de Agosto de 2017 | Aceptado: 1 Septiembre de 2017 | Publicado en línea: Julio-Diciembre de 2017 | 


\title{
Autoría y Derechos de Propiedad Intelectual
}

\section{EL QUEHACER DEL PROFESIONAL DE LA PSICOLOGÍA DE LA SALUD: DEFINICIONES Y OBJETIVOS}

\author{
Laura Edith Pérez-Laborde y Liliana Moreyra-Jiménez \\ FES Iztacala, UNAM \\ México
}

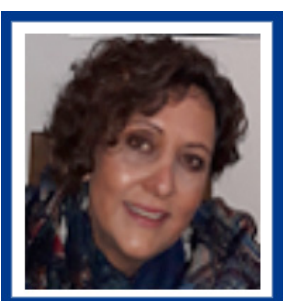

Laura Edith Pérez-Laborde

FES Iztacala, UNAM

Correo: edith.perez@ired.unam.mx

Licenciada y maestra en Psicología por la UNAM con residencia en terapia familiar sistémica. Profesora de asignatura en la licenciatura en Psicología a Distancia (SUAYED) en el área de Psicología de la Salud, jefa de Claustro en Procesos en Psicología de la Salud, docente y supervisora en la maestría en Psicología, Ver más...

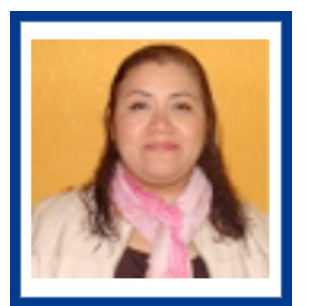

Liliana Moreyra-Jiménez FES Iztacala, UNAM

Correo: liliana.moreyra@ired.unam.mx

Profesora de la licenciatura en Psicología a Distancia en la Facultad de Estudios Superiores Iztacala (SUAyED-UNAM) de las áreas en Psicología de la Salud y Psicología Clínica. Licenciada en Psicología por la Facultad de Psicología de la UNAM.

Ver más...

\section{CONTRIBUCIÓN DE LAS AUTORAS}

Laura Edith Pérez-Laborde coordinó la integración del documento, construyó el esquema y participó en la redacción del artículo, I Liliana Moreyra-Jiménez participó en la redacción del artículo

\section{AGRADECIMIENTOS}

[Agradecimiento por parte de los autores a intituciones, personas, etc. participantes en el estudio.]

\section{DATOS de FiLIACIÓN DE LAS Autoras}

Sistema de Univeridad Abierta y Educación a Distancia, Facultad de Estudios Superiores Iztacala, UNAM.

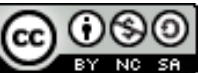

Copyright: @ 2017 Pérez-Laborde, L. E. \& Moreyra-Jiménez, L.

Este es un artículo de acceso abierto distribuido bajo los términos de la licencia Creative Commons Reconocimiento-NoComercial-Compartirlgual 4.0 Internacional, por lo que su contenido gráfico y escrito se puede compartir, copiar y redistribuir total o parcialmente sin necesidad de permiso expreso de sus autoras con la única condición de que no se puede usar con fines directamente comerciales y los términos legales de cualquier trabajo derivado deben ser los mismos que se expresan en la presente declaración. La única condición es que se cite la fuente con referencia a la Revista Digital Internacional de Psicología y Ciencia Social y a sus autoras. 


\section{TABLA DE CONTENIDO}

INTRODUCCIÓN

MÉTODO

Diseño., 6

Participantes., 6

Instrumento., 7

Procedimiento., 7

Resultados, 7

CONCLUSIONES

Discusión

REFERENCIAS

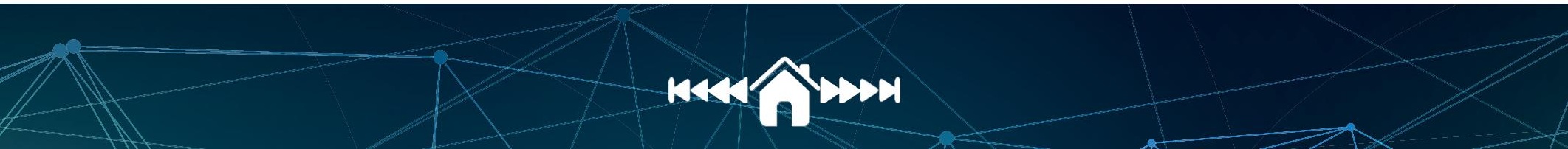




\section{INTRODUCCIÓN}

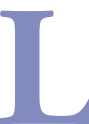

a salud había sido entendida, durante muchos siglos, como la ausencia de enfermedad, pero en 1974 la Organización Mundial de la Salud (OMS) declaró que esta forma de concebirla limitaba la comprensión acerca de su complejidad y su abordaje, de modo que la definió desde entonces como "el completo bienestar físico, mental y social y no simplemente como la ausencia de dolencias o enfermedades" (Oblitas, 2008: 222). Esta nueva concepción de la salud impulsó no sólo un cambio conceptual, sino que abrió la perspectiva respecto a los retos profesionales que implicaba.

La salud había sido entendida, durante muchos siglos, como la ausencia de enfermedad, pero en 1974 la Organización Mundial de la Salud (OMS) declaró que esta forma de concebirla limitaba la comprensión acerca de su complejidad y su abordaje, de modo que la definió desde entonces como "el completo bienestar físico, mental y social y no simplemente como la ausencia de dolencias o enfermedades" (Oblitas, 2008: 222). Esta nueva concepción de la salud impulsó no sólo un cambio conceptual, sino que abrió la perspectiva respecto a los retos profesionales que implicaba.

La psicología de la salud es un campo de conocimiento de la Psicología que comenzó su desarrollo en la década de los setenta como respuesta ante la necesidad de abordar el proceso de salud-enfermedad de los seres humanos desde una nueva visión integradora respecto a los elementos biológicos, psicológicos y sociales que intervienen en ella, colaborando con otras disciplinas científicas en su esfuerzo por mejorar la calidad de vida y disminuir la exposición ante diversos riesgos para la salud, específicamente los que provienen de factores psicológicos, emocionales, cognitivos, conductuales y relacionales del propio individuo (Oblitas, 2008).

Este campo ha avanzado notablemente respecto a la construcción de sus propios modelos, la comprensión de los aspectos involucrados en el comportamiento saludable y no saludable, así como las estrategias de intervención que la diferencian de otras áreas de la Psicología; sin embargo, en la práctica aún falta camino por recorrer respecto a la comprensión de lo que se aporta en el campo profesional.

Piña, Sánchez, García, Ybarra y García (2013) señalan tres aspectos que aún representan un reto para el profesional de la Psicología. El primero, fundamental, es el hecho de que la psicología de la salud no está siendo considerada en los marcos normativos en materia de atención a la salud, ya que se considera un asunto de competencia médica casi exclusivamente; el segundo es que los esfuerzos para intervenir de manera interdisciplinaria se consolidan desde las instituciones de salud, colocando a la medicina como el eje de las acciones en las que la prevención tiene poco o nulo lugar. El tercer aspecto es que la formación académica del profesional que interviene desde la psicología de la salud, salvo algunas excepciones, aún es deficiente y no proporciona los conocimientos teórico-metodológicos suficientes ni entrena las competencias necesarias para hacer intervenciones congruentes con los objetivos y herramientas de esta área.

En una revisión de planes de estudio de la licenciatura en Psicología en México (Universidad Autónoma Metropolitana, Universidad Iberoamericana, Universidad del Valle de México y Universidad Autónoma de Puebla, por mencionar algunos) se observa que no todos los programas contemplan el área de psicología de la salud, y en algunos casos, como en los programas presenciales de las diferentes facultades de la Universidad Nacional Autónoma de México (UNAM), se considera como área clínica y de la salud; sólo la licenciatura a distancia contiene en su plan de estudios el área de psicología de la salud como un campo de profundización (UNAM, 2012). La Universidad de Sonora, en cambio, ofrece una licenciatura en Psicología de la Salud, y la Universidad de Guadalajara tiene un programa de maestría en esta área. En Cuba se incluye como una asignatura en la carrera de medicina.

Lo anterior resulta relevante porque la mayoría de los psicólogos insertos en instituciones donde cumplen funciones propias de la psicología de las salud no tienen formación académica en esta área, o su formación no les dota de suficientes competencias específicas (Piña et al., 2013).

También se ha observado que quienes se encuentran trabajando en este campo no se definen a sí mismos como psicólogos de la salud ni identifican sus intervenciones como acciones propias de este campo. En otros casos se percibe una gran confusión respecto a qué acciones se pueden realizar para responder a los objetivos de la psicología de la salud y con qué estrategias, de modo que, aún teniendo la intención de efectuar una intervención en esta área, se hace en realidad desde otro dominio de la Psicología (Piña et al., 2013).

Además, las instituciones de salud en México contratan profesionales de la Psicología con formación clínica, en tanto la Norma Oficial Mexicana (2015) ${ }^{1}$ así lo establece

1 La Secretaría de Salud (2015) crea la Norma Oficial Mexicana NOM-025-SSA2-2014, para la prestación de servicios de salud en unidades de atención integral hospitalaria médico-psiquiátrica que define la atención psicológica como: 4.1.4 Atención Psi- 
al describir la atención psicológica, aunque en el perfil se incluyen acciones propias de la psicología de la salud.

Entonces si una intervención tiene un efecto positivo en la persona o grupo con el que se trabaja podría no ser importante mezclar la psicología clínica con la psicología comunitaria y la medicina conductual; sin embargo, no puede considerarse que eso resulte productivo en lo que respecta al desarrollo teórico metodológico de esta área y a la comprensión de sus objetivos y estrategias particulares; mucho menos para clarificar el perfil profesional que, en términos de competencias, debería tener (Arco \& Fernández, 2002; Piña et al., 2013).

Bajo esta perspectiva se pretende clarificar los elementos que delimitan el quehacer específico del profesional de la psicología de la salud en los diversos contextos donde se inserta y el enfoque de las intervenciones que realiza que la definen como tal.

\section{Objeto de estudio de la psicología de la}

\section{SALUD}

A lo largo de los últimos años se han escrito diversas definiciones de psicología de la salud, cada una de ellas enfatizando aspectos distintos; se muestran aquí las que se consideran más representativas de la forma como se entiende actualmente el objeto de estudio de esta área.

La psicología de la salud es el campo de especialización de la psicología que centra su interés en el ámbito de los problemas de salud, especialmente físicos y orgánicos, con la principal función de prevenir la ocurrencia de los mismos o de tratar o de rehabilitar éstos en caso de que tengan lugar, utilizando para ello metodología, los principios y conocimientos de la actual psicología científica sobre la base de que la conducta constituye, junto con las causas biológicas y sociales, los principales determinantes tanto de la salud como de la mayor parte de las enfermedades y problemas humanos de la salud existentes en la actualidad (Carrobles, 1993, apud Gómez, 2007: 163).

La suma de contribuciones profesionales, científicas y educativas específicas de la psicología como disciplina, para la promoción y mantenimiento de la salud, la prevención y tratamiento de la enfermedad, la identificación de los correlatos etiológicos y diagnósticos de la salud, la enfer-

cológica: Es la atención especializada brindada por un psicólogo clínico que trata diferentes trastornos mentales y del comportamiento y problemáticas de salud mental utilizando psicoterapia y otros recursos terapéuticos dependiendo de la severidad de la patología. La atención psicológica difiere de acuerdo al tipo de unidad de atención de salud mental, si es de primer nivel donde se hacen detecciones de casos, psicoeducación y actividades preventivas; las unidades de segundo nivel atienden casos de forma ambulatoria, el tercer nivel atiende a personas que están en hospitalización o en alguna unidad comunitaria de rehabilitación psicosocial. medad y la disfunción asociada, además del mejoramiento del sistema sanitario y la formulación de una política de la salud (Matarazzo, 1980, apud Labiano, 2010: 15).

Un conjunto de contribuciones científicas y profesionales (investigación, evaluación, intervención, formación y gestión) de la psicología relativas a la promoción y acrecentamiento de la salud y a la prevención, tratamiento y rehabilitación de la enfermedad, así como análisis y mejora del sistema sanitario y de las políticas de salud, y consistentes en la investigación de la importancia de los aspectos comportamentales en la preservación de la salud (y generación, mantenimiento o eliminación de la enfermedad) y la elaboración y aplicación de programas destinados al control o modificación de los mismos (Godoy, 1999, apud Gómez, 2007: 165).

Las autoras coinciden con la definición de Gómez (2007) ya que sintetiza todos los elementos implicados en el campo de la Psicología de la Salud y señala las acciones profesionales con que se puede intervenir. Es así que esta área surgió como una necesidad de abordaje de algunos aspectos de la salud y de los procesos de enfermedad de las personas, aporta también elementos para mejorar el sistema sanitario que no estaban considerados por el modelo biomédico, ya que éste ha dirigido su atención hacia procesos biofísicos de la enfermedad y ha ignorado los aspectos psicológicos y sociales que inciden en ella, para dar prioridad a la visión curativa sobre la preventiva y enfocar sus intervenciones en la prolongación de los años de vida y no en la calidad de la misma.

En la década de los setenta surgió el modelo biopsicosocial como alternativa al biomédico e introdujo una mirada ampliada respecto a las necesidades y retos individuales, institucionales y sociales relativos a la salud. Desde el campo de la Psicología de la Salud, que adopta la visión del modelo biopsicosocial, se ofrecen nuevas explicaciones y alternativas de intervención ante el aumento de enfermedades crónico-degenerativas así como una comprensión más amplia de la responsabilidad de cada persona en el desarrollo de las enfermedades que padece y el modo en que las afronta (Grau \& Hernández, 2005).

El modelo biomédico se sustenta en la idea de que se tiene salud cuando no se padece ninguna enfermedad; el modelo biopsicosocial plantea que tanto la salud como la enfermedad son procesos influenciados por aspectos biológicos, psicológicos y sociales, lo que propone que la salud se encuentra en estrecha relación con el bienestar experimentado por un individuo en la esfera biológica, psicológica y social, y más recientemente con la calidad de vida; ambos conceptos aluden a aspectos subjetivos que provienen del propio individuo, asumiendo que están vinculados directamente con su estado de salud física (Juárez, 2011). 
Desde este nuevo enfoque se cuestiona el papel pasivo y receptor de la persona enferma, tanto en lo que concierne a su padecimiento como en su tratamiento. Incluso podría afirmarse que estas formas de comprender la salud y la pérdida de ésta visualizan al individuo integralmente, en interacción, de forma sistémica, con todas las esferas que le rodean y conforman. El modelo biopsicosocial sustenta esta visión, contemplando que existen múltiples factores que inciden en el estado de salud, en su deterioro y su recuperación, de modo que no debe esperarse a que una persona enferme para atenderlo porque se pueden generar acciones de prevención, si se comprenden y abordan las variables involucradas que, de manera amplia y de acuerdo con Labiano (2010) son las siguientes.

1. Situacionales: factores que incrementan, disminuyen o eliminan conductas saludables, como son las redes de apoyo, el acceso a la información, los factores económicos y los modelos sociales de comportamiento relacionado con la salud, tanto del entorno inmediato como los promovidos culturalmente.

2. Personales: factores que influyen en la aceptación y sostenimiento de patrones saludables a partir de las creencias, la motivación y las expectativas.

3. Consecuencias anticipadas: se refieren a la percepción subjetiva de la persona acerca de los costos o beneficios que conlleva la adopción de conductas saludables y la evaluación que hace a partir de dicha percepción.

\section{Objetivos de intervención en psicología de}

\section{LA SALUD}

Así, se plantea que el objeto de estudio de la psicología de la salud es la comprensión de los factores psicológicos involucrados en el proceso de salud-enfermedad de un individuo o una comunidad y el objetivo principal en cualquier intervención será la prevención. Entendida en su más amplio significado, la prevención, aplicada a este campo del conocimiento, estará dirigida a optimizar las posibilidades de las personas para desarrollar un adecuado estado de salud y para afrontar la enfermedad del mejor modo que sea posible, así como la adherencia al tratamiento prescrito desde otras áreas de atención a la salud (medicina, nutrición y rehabilitación, entre otras), además de mejorar la calidad de vida.

La división 38 de la American Psychological Association (APA) plantea que, además, la psicología de la salud tiene como objetivos aquellos relacionados con comprender y evaluar el estado de bienestar físico y los diferentes factores biológicos, psicológicos y sociales involucrados en el pro- ceso de salud-enfermedad, así como entender los métodos de investigación psicológica aplicados a la prevención y la intervención ante la enfermedad (Gómez, 2007).

Sin embargo, la prevención puede ser abordada desde dos perspectivas distintas, ambas con el objetivo de mejorar la calidad de vida de las personas; Oblitas (2008) las describe de la siguiente manera.

a) Promoción de la salud: Impulsar estilos de vida saludables con la intención de desarrollar un estado integral de bienestar físico y psicológico en el individuo y así mejorar su calidad de vida. De algún modo la promoción de la salud busca optimizar las posibilidades de una persona o grupo para lograr un desarrollo pleno y la mejor calidad de vida que puede tener de acuerdo con sus condiciones particulares. Como tal, es una estrategia establecida en Ottawa, en 1986, donde se la define como: "el proceso que proporciona a los individuos y las comunidades los medios necesarios para ejercer un mayor control sobre su propia salud y así poder mejorarla" (Vignolo, Vacarezza, Álvarez \& Sosa, 2011: 13). Es decir, es una estrategia basada en la idea de que las personas pueden desarrollar comportamientos que optimizan su salud si se proporcionan herramientas que le permitan tener control y dirección. Al promover la salud, se hace énfasis en la comprensión sobre los aspectos que la influyen positivamente y que generan bienestar.

b) Prevención de la enfermedad: Esta estrategia había sido la más usada dada la influencia del modelo biomédico en la atención del proceso salud enfermedad. Las acciones que plantea se basan en la intervención directa sobre los factores de riesgo asociados con el desarrollo de una enfermedad, intentando minimizar o eliminar su influencia, así que la intención es modificar hábitos no saludables y eliminar comportamientos de riesgo asociados a múltiples patologías crónicas, agudas, infecciosas y accidentes que pueden evitarse o retrasarse si se adoptan las medidas pertinentes.

De manera que la prevención, desde sus dos perspectivas, es el objetivo principal de la psicología de la salud, pero se plantean otros dos, implícitamente. El primero - dirigido al individuo o la comunidad — se refiere a la evaluación y tratamiento de los aspectos psicológicos (conducta, emociones y pensamientos) relacionados con enfermedades específicas como diabetes, intestino irritable, cáncer, SIDA, hipertensión, dependencia a sustancias y epidemias, entre otras. El segundo, que coloca su enfoque en el funcionamiento institucional y político, planteando la evaluación y mejora del sistema sanitario mediante propuestas dirigidas a optimizar la atención sanitaria en lo que se refiere a la práctica profesional y los programas 
preventivos dirigidos a la población, así como las políticas públicas relacionadas con dichos programas.

Este objetivo resulta muy relevante puesto que los programas preventivos y las políticas públicas que los sustentan deben estar basados en información científica que guíe las acciones a partir de la comprensión de las variables en las que se debe intervenir, los modelos teóricos y estrategias que podrían ser más efectivos; de otro modo, se realizan acciones que terminan siendo inútiles $y$, desde luego, no preventivas. Al respecto, Arco y Fernández (2002) señalan que las fallas en la efectividad de los programas diseñados para la prevención se vinculan sobre todo con la falta de sustento teórico, el hecho de no considerar la necesidad de la colaboración interdisciplinaria, la dificultad para definir los grupos de riesgo y la ineficacia de la evaluación de resultados, por no decir inexistencia. Las políticas públicas no contemplan la necesidad de incorporar al profesional de la psicología de la salud en el diseño de estrategias y, en muchos casos, tampoco en las acciones, ya que la práctica está regulada por el Sistema de Salud y la prioridades que desde ahí se establecen.

Tanto la promoción de la salud como la prevención de la enfermedad deben ser entendidas como formas de enfocar una intervención que, al mismo tiempo, guían las estrategias para efectuarlas. Resulta de suma relevancia distinguirlas claramente porque el diseño de las intervenciones debe ser congruente con ellas; ambas responden al objetivo primordial de hacer prevención, pero desde diferentes perspectivas.

Para Reynoso y Seligson (2005) la prevención es fundamental, ya que actualmente existe evidencia epidemiológica que permite identificar condiciones de riesgo ante las cuales es factible establecer programas preventivos. La OMS (1998, apud Vignolo et al., 2011) define prevención como "Medidas destinadas no solamente a prevenir la aparición de la enfermedad, tales como la reducción de factores de riesgo, sino también a detener su avance y atenuar sus consecuencias una vez establecidas" (p. 12).

De acuerdo con Vignolo et al. (2011), la prevención debe comprender el proceso de la enfermedad y sus múltiples componentes para lograr incidir en ésta y diseñar sus acciones partiendo del nivel en que se pretende realizar la intervención, ya sea para intentar evitarla, para detectarla oportunamente o para minimizar sus secuelas.

Dichos niveles de prevención, desde los que se puede intervenir, han sido retomados de los propuestos por la psicología social y se han clasificado de la siguiente forma (Martínez \& García, 2012; Santacreu, Marquéz \& Rubio, 1997; Vingolo et al., 2011).

- Prevención primaria. Parte de la idea de que educar es prevenir; su objetivo es evitar la enfermedad identificando los factores causales para incidir en ellos antes de que se presente el problema; la intervención se produce con la intención de evitarlo.

- Prevención secundaria. Intenta detectar y aplicar tratamientos a las enfermedades en sus primeros estadios. Está dirigida al diagnóstico oportuno y enfocada a reducir al mínimo una alteración con base en la detección temprana y el tratamiento adecuado, esenciales para el control de una enfermedad o para superarla. La intervención preventiva de nivel secundario es ideal cuando aún el daño al organismo no está tan avanzado y se puede detener o controlar.

- Prevención terciaria. Tiene lugar cuando ya se ha diagnosticado la enfermedad, pero empieza a manifestarse e intenta evitar su avance, así como sus complicaciones. Se busca reducir las consecuencias de una alteración o enfermedad. Podría definirse como una acción de tratamiento o rehabilitación después de haber experimentado un problema de salud que ya está presente; puede empeorar y se previenen estados más avanzados de la enfermedad. Este tipo prevención pretende trabajar con las consecuencias que un problema puede ocasionar y evitar que se agrave.

- Prevención cuaternaria. Se ha denominado así a las acciones dirigidas a evitar procedimientos y medicación innecesarios o excesivos; se enfoca en el empoderamiento del paciente y sus familiares respecto a su derecho a decidir respecto al tratamiento ante su enfermedad; también en este nivel se ubican las acciones del sistema sanitario dirigidas a evaluar sus protocolos de atención con pacientes terminales o en proceso de muerte inminente, y el suministro de medicamentos con graves efectos secundarios. Aunque no existe consenso en cuanto a la necesidad de considerar este nivel de prevención, en años recientes se ha manifestado la preocupación de muchos profesionales en cuanto a los efectos negativos de la intervención médica en la que se ha priorizado la realización de procedimientos invasivos y el suministro de medicamentos ante problemas de salud que podrían tratarse de otras formas y que tienen efectos secundarios que impactan en la calidad de vida de la persona (Gervás, 2006).

Para clarificar los primeros tres niveles de prevención, en la tabla 1 se muestran acciones específicas desde las perspectivas descritas, prevención de la enfermedad y promoción de la salud, que pueden usarse en cada uno de los niveles, pero se dirigen a diferente población. Como se observa en la tabla, la perspectiva de promoción de la salud se utiliza cuando lo que se pretende es propiciar el desarrollo de la persona o la comunidad, para con ello mejorar su calidad de vida, tanto en el caso de que ya haya 
enfermado como cuando se encuentra sano. El enfoque de la prevención de la enfermedad se utilizará cuando lo que se desea es modificar un comportamiento que ya se ha descrito como no saludable o de riesgo para la salud.

\section{Tabla 1.}

Intervención en los tres niveles de prevención desde las perspectivas de la promoción de la salud y la prevención de la enfermedad.

\begin{tabular}{|c|c|c|}
\hline & $\begin{array}{c}\text { Promoción DE LA } \\
\text { SALUd }\end{array}$ & $\begin{array}{c}\text { Prevención de LA } \\
\text { enfermedad }\end{array}$ \\
\hline $\begin{array}{c}\text { Perspectiva/ } \\
\text { Nivel }\end{array}$ & $\begin{array}{l}\text { Su objetivo es } \\
\text { fomentar hábitos de } \\
\text { salud a través de: } \\
\text { - La reflexión } \\
\text { respecto a las } \\
\text { creencias. } \\
\text { - Proporcionar } \\
\text { información. } \\
\text { - Fomento de } \\
\text { habilidades } \\
\text { específicas. }\end{array}$ & $\begin{array}{l}\text { Su objetivo es } \\
\text { modificar hábitos no } \\
\text { saludables y eliminar } \\
\text { comportamientos de } \\
\text { riesgo. }\end{array}$ \\
\hline
\end{tabular}

Población general que no presenta un riesgo específico

Ejemplo:

- Entrenamiento en

habilidades asertivas, manejo emocional

o sexualidad

responsable dirigido a adolescentes.

Prevención PRIMARIA

Prevención SECUNDARIA
- Informar respecto

a las consecuencias

individuales,

familiares y físicas de

la adicción al alcohol.

- Campaña

informativa acerca de

habilidades de crianza

de niños y niñas

respecto al manejo

de sus hábitos

alimenticios.

Población en situación de riesgo o vulnerabilidad

- Taller sobre uso de condón para prevenir $\mathrm{VIH}$, dirigido a adolescentes.

Ejemplo:

- Taller o intervención individual o familiar dirigidos a modificar las creencias que

Ejemplo:

- Entrenamiento en prácticas de sexo seguro a parejas de personas infectadas con VIH.

\begin{tabular}{|c|c|c|}
\hline \multirow[b]{2}{*}{$\begin{array}{l}\text { Perspectiva/ } \\
\text { Nivel }\end{array}$} & $\begin{array}{c}\text { Promoción DE LA } \\
\text { SALUD }\end{array}$ & $\begin{array}{l}\text { Prevención de LA } \\
\text { enfermedad }\end{array}$ \\
\hline & $\begin{array}{l}\text { Su objetivo es } \\
\text { fomentar hábitos de } \\
\text { salud a través de: } \\
\text { - La reflexión } \\
\text { respecto a las } \\
\text { creencias. } \\
\text { - Proporcionar } \\
\text { información. } \\
\text { - Fomento de } \\
\text { habilidades } \\
\text { específicas. }\end{array}$ & $\begin{array}{l}\text { Su objetivo es } \\
\text { modificar hábitos no } \\
\text { saludables y eliminar } \\
\text { comportamientos de } \\
\text { riesgo. }\end{array}$ \\
\hline \multirow[b]{2}{*}{$\begin{array}{l}\text { PreVención } \\
\text { TERCIARIA }\end{array}$} & \multicolumn{2}{|c|}{ Población con un diagnóstico de enfermedad } \\
\hline & $\begin{array}{l}\text { Ejemplo: } \\
\text { - Fomento de } \\
\text { la comunicación } \\
\text { asertiva, } \\
\text { entrenamiento del } \\
\text { manejo de emociones } \\
\text { de la persona enferma } \\
\text { respecto a su familia. }\end{array}$ & $\begin{array}{l}\text { Ejemplo: } \\
\text { - Taller o intervención } \\
\text { individual o familiar } \\
\text { acerca de técnicas de } \\
\text { manejo de ansiedad } \\
\text { o biofeedback } \\
\text { con pacientes } \\
\text { hipertensos. }\end{array}$ \\
\hline
\end{tabular}

\section{¿Cómo SE hace UNA INTERVENCIÓN DESDE LA Psicología de la Salud?}

De acuerdo con García, Piqueras, Rivero, Ramos y Oblitas (2008), Morales (1997) y Stone (1998), el profesional de la psicología de la salud inicia su intervención realizando una evaluación que consiste en la recopilación de la información necesaria que le permita comprender los factores psicológicos involucrados en el problema en que pretende incidir. Para efectuar la evaluación puede utilizar diversas herramientas, entre las que se encuentran algunas escalas validadas de acuerdo con tipo de enfermedad o problema de salud —entrevistas, observaciones directas, registros psicofisiológicos y bioquímicos-, que finalmente se conforman como punto de partida para elaborar un diagnóstico; además, puede utilizar información epidemiológica que le permita comprender la prevalencia de enfermedades, características de la población, etcétera.

A partir de los resultados de la evaluación (análisis e interpretación de resultados), se realiza un proceso de análisis de las características del problema, se efectúa un diagnóstico y se diseña la intervención. Tanto el diagnóstico como la intervención deben partir de los postulados específicos del modelo de salud que se haya elegido, así como las variables en que incidirá. Sin embargo, las herramientas específicas provienen de cualquier modelo teórico de la Psicología, así que puede hacerse una intervención con técnicas de modificación de conducta, grupos de reflexión y/o psicoeducación, entre otros. 
Los modelos a los que se hace referencia han sido construidos desde diferentes teorías intentando explicar, predecir e intervenir ante los aspectos psicológicos vinculados con el proceso de salud-enfermedad; cada uno ofrece diferentes explicaciones y postula las variables en las que es necesario incidir para prevenir enfermedades (Enríquez, Sánchez \& Robles, 2011). Aunque se han desarrollado muchos, a continuación se mencionan algunos de ellos, así como sus variables.

- Modelo de creencias en salud, creado por Hackbaum, Kegels, Leventhal y Rostenstock en 1950, considera que los comportamientos relacionados con la salud son el resultado de las creencias y valoraciones internas que hacemos las personas, por lo que, para prevenir enfermedades, los programas deben apuntar hacia cuatro variables (que son la susceptibilidad, los beneficios, las barreras y la severidad) que una persona percibe respecto a comportamientos saludables y la posibilidad de enfermar (Cabrera, Tascón \& Lucumí, 2001).

- Modelo de autoeficacia, creado por Bandura a mediados de los noventa, plantea que la capacidad percibida por la persona respecto a su posibilidad de ejecutar exitosamente un comportamiento preventivo (autoeficacia percibida) es la variable en que es necesario incidir en los programas de prevención, ya que este aspecto se ha observado como un importante predictor del comportamiento (Bandura, 1977).

- Modelo transteórico. En 1970 Prochaska creó un programa de tratamiento para personas con adicciones utilizando principios de diversas teorías; en la década de los noventa consolidó su teoría planteando que el cambio conductual relacionado con hábitos no saludables o que ponen en riesgo la salud, es un proceso en etapas; que las personas tienen diferentes niveles de motivación y de intención respecto al cambio, por lo que para optimizar la intervención es necesario identificar en qué etapa se encuentra la persona para enfocar las intervenciones en lo que necesita para pasar a la siguiente teniendo en cuenta además, como variables, la tentación, la autoeficacia y algunas variables psicosociales como intermediarias del cambio y los procesos experienciales (Prochaska, DiClemente \& Norcross, 1994) Este modelo no es explicativo respecto a las variables que afectan el comportamiento, como los dos anteriores, sino que se centra en el cambio conductual (Cabrera, 2000).

- Teoría de acción razonada y teoría de la conducta planeada. En 1975 Fishbein y Azjen desarrollaron una teoría psicológica que intentaba explicar que el comportamiento humano está determinado por la intención de ejecutarla. Ésta depende de la actitud individual hacia ese comportamiento y está mediada por la percepción de lo que piensan los otros, así como por la motivación para satisfacer esas expectativas, variable que fue nombrada como norma subjetiva. A mediados de los ochenta Azjen replanteó la teoría, a la que denominó de la conducta planeada, afirmando que además de las variables propuestas en la teoría de acción razonada era necesario contemplar otra que es el control conductual percibido, entendido como lo que la persona cree que es capaz de hacer para cuidarse a sí mismo y, de ese modo, prevenir (Azjen, 2011). Aunque estas dos teorías surgieron inicialmente como una explicación de los aspectos que determinan el cambio de conducta, las variables que proponen se han usado como guía en acciones de prevención ante adicciones y salud sexual.

- Modelo de salud biológica propuesto por Emilio Ribes a finales de los ochenta es un modelo explicativo-investigativo basado en los principios teóricos del interconductismo; plantea que el grado de enfermedad que puede desarrollar una persona depende de qué tan agresiva sea ésta a nivel biológico y de su grado de vulnerabilidad, que es el resultado de múltiples factores propios de la interacción del individuo —entendido como sujeto bio-psico-social- con su ambiente (Piña, 2008). En este modelo se afirma que un comportamiento preventivo será eficaz si existen competencias efectivas en la comprensión y evaluación del riesgo, así como condiciones suficientes para lograrlo en términos de sus propias habilidades y el tipo de ambiente en que desenvuelve. De modo que un programa efectivo, de acuerdo con este modelo, debe contemplar dicha interacción individuo-ambiente (Bayés \& Ribes, 1989).

- Modelo de información, motivación y habilidades conductuales propuesto por Fisher y Fisher en 1992, es un modelo de cambio conductual que afirma se pueden desarrollar conductas preventivas cuando: a) se tiene información suficiente y relevante; b) existe motivación para hacerlo a partir de las creencias personales, la norma subjetiva, la autoeficacia y la intención, y c) se aprenden habilidades específicas para modificar la condición de riesgo (Fisher \& Fisher, 2000).

- Modelo integral desarrollado por Fishbein a principios de este siglo; de acuerdo con Enríquez et al. (2011), retoma elementos de algunos de los modelos mencionados, planteando que la intención de cambio está influida por las habilidades conductuales del 
sujeto y por los límites ambientales que se entienden como las características del sujeto influidas por variables que el autor denominó distales, como edad, lugar de residencia, nivel socioeconómico, aspectos culturales, estereotipos, personalidad, manejo de emociones, autoeficacia y su exposición a intervenciones (programas de prevención, información).

Tabla 2.

Acciones y competencias requeridas del profesional de la Psicología de la Salud.

\begin{tabular}{|c|c|c|}
\hline Acciones & Objetivos & Competencias \\
\hline Diagnóstico & $\begin{array}{l}\text { Observación, } \\
\text { evaluación y } \\
\text { comprensión de } \\
\text { los elementos } \\
\text { psicológicos } \\
\text { y del entorno } \\
\text { involucrados en un } \\
\text { problema de salud. }\end{array}$ & $\begin{array}{l}\text { - Conocimientos de } \\
\text { Psicología general } \\
\text { y su aplicación ante } \\
\text { problemas de salud. } \\
\text { - Comprensión de la } \\
\text { dimensión psicológica } \\
\text { involucrada en la } \\
\text { salud. } \\
\text { - Manejo de los } \\
\text { modelos teóricos } \\
\text { propios de la } \\
\text { Psicología de la Salud. } \\
\text { - Conocimiento de } \\
\text { políticas públicas y } \\
\text { reglamentaciones en } \\
\text { materia de salud. } \\
\text { - Habilidad para } \\
\text { evaluar necesidades } \\
\text { individuales, } \\
\text { familiares o } \\
\text { comunitarias. } \\
\text { - Habilidad para } \\
\text { evaluar resultados } \\
\text { de intervenciones } \\
\text { realizadas ya sea } \\
\text { en programas } \\
\text { preventivos de } \\
\text { nivel primario, } \\
\text { secundarias, terciarias } \\
\text { o cuaternarias. }\end{array}$ \\
\hline
\end{tabular}

Modificar aspectos psicológicos (cognitivos, emocionales $\mathrm{y} / \mathrm{o}$ conductuales) $\mathrm{y} / \mathrm{o}$ del entorno social que interfieren en la adecuada adherencia al tratamiento, el afrontamiento de la enfermedad y el desarrollo de conductas que optimizan la calidad de vida posible.

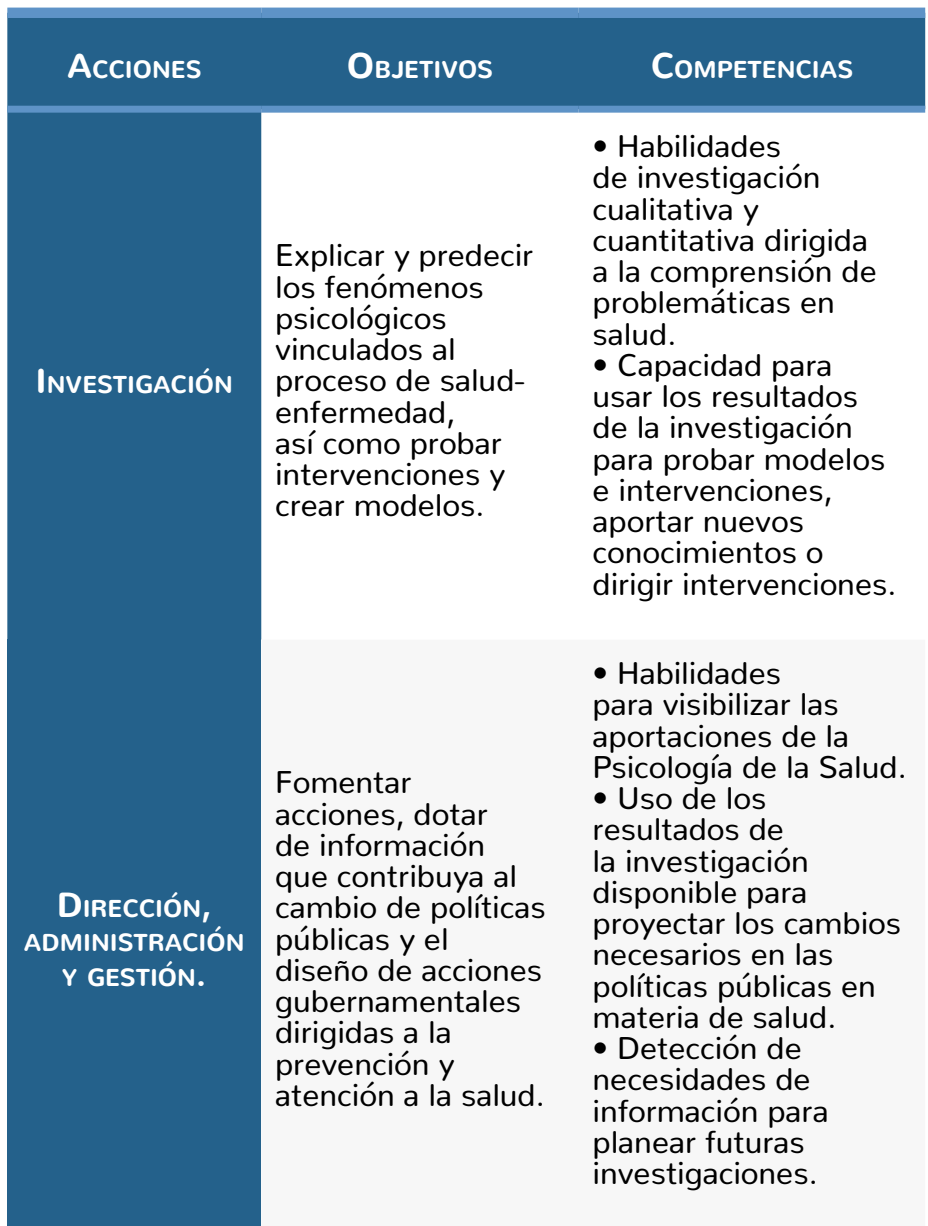

Resumiendo lo que se escribe en este apartado, se tiene que, desde el campo de la psicología de la salud, las intervenciones se refieren a programas de prevención primaria, secundaria o terciaria, de modo que las acciones se diseñan para promover la salud o prevenir la enfermedad con población general, en situación de riesgo o para acompañar procesos de enfermedad dirigidos a la adherencia al tratamiento con población que ya ha desarrollado algún problema de salud; éstos pueden ser individuales, grupales, familiares y comunitarios. En la tabla 2 se muestran los objetivos y competencias requeridas en las acciones que se realizan desde la psicología de la salud de acuerdo con las que señalan Piña et al. (2013).

En cuanto a los lugares para realizar una intervención, podrían ser centros de salud y hospitales, centros geriátricos, unidades de rehabilitación, unidades de dolor crónico, cuidados paliativos y atención a enfermos terminales, centros de voluntariado u organizaciones no gubernamentales, unidades de salud laboral, sector salud público y privado, instituciones de educación pública y privada, comunidades urbanas y rurales. En la práctica privada se estará haciendo una intervención desde 
la psicología de la salud cuando el motivo de consulta se relacione con el aspecto físico de la salud, el cual puede ser mantenerla, evitar una enfermedad o mejorar la calidad de vida (García et al., 2008). Algunos ejemplos de ello podrían ser el manejo de ansiedad que impide la adecuada adherencia a un programa de control de peso, superar alguna adicción, conflictos familiares que interfieren con el adecuado afrontamiento de problemas físicos de salud de alguno de sus integrantes, o la depresión que obstaculiza la toma de decisiones acerca del tratamiento de una enfermedad crónica.

Así que los problemas de salud en los que se puede intervenir son muy diversos; algunos de ellos son: enfermedades crónico-degenerativas, enfermedades infecciosas, trastornos alimentarios, adicciones, estrés, dolor, accidentes. Lo que es importante destacar es que la psicología de la salud está enfocada en lograr mejorar la salud física, bienestar y la calidad de vida que se relaciona con ésta, incidiendo en los factores psicológicos que obstaculizan dicho objetivo.

\section{CAMPo de ACCIÓN del PROFESIONAL EN LA PSI-} COLOGÍA DE LA SALUD

La psicología de la salud es un campo que aún está en desarrollo, y en muchos países, incluyendo México, como se comentaba, no está todavía claramente diferenciada de otras áreas en los programas de formación y en las funciones que desempeña el profesional de la Psicología en las instituciones y programas de salud. Independientemente de si el propio profesional de la psicología de la salud se define como tal o el campo de trabajo donde está inserto lo identifica, es la función que cumple y el objetivo con que lo hace lo que, a final de cuentas, lo coloca en este campo (Morales, 1999; Piña \& Rivera, 2006).

Por otra parte, la intervención desde la psicología de la salud — puede ser de dos tipos, activa o pasivaes siempre de tipo activo, ya que está diseñada para lograr la modificación del comportamiento no saludable, a diferencia de otras ciencias cuyo enfoque es la modificación del entorno para reducir el riesgo, lo cual se ha identificado como de tipo pasivo.

Para clarificar cuáles se consideran intervenciones pasivas y cuáles activas, se mencionará el ejemplo de las políticas públicas que recientemente dirigieron sus esfuerzos hacia la prevención de riesgos relacionados con el tabaquismo (The Association of Faculties of Medicine of Canada, 2012). La legislación ha señalado los espacios que deben ser "libres de humo" y establece una sanción para quien fume o permita fumar en ellos; esta acción protege a quienes no fuman y tiende a modificar directamente el comportamiento del fumador, en términos de no poder fumar en ciertos espacios, pero no está dirigida a incidir en el consumo de tabaco como tal, así que, en tanto esta acción se dirige al cambio del entorno del fumador, se considera de tipo pasivo.

En tanto que una intervención activa puede ir desde proporcionar información hasta la aplicación de una técnica de modificación de conducta, siempre con la intención de lograr un cambio directamente en la población hacia quien está dirigida la intervención.

De manera que los ambientes en que el profesional de la psicología de la salud se puede insertar son muy variados, pero resulta útil contextualizarlos de acuerdo con la siguiente clasificación.

- Ambientes institucionalizados. Actividades que se desprenden de los objetivos de la institución, plan de desarrollo o ejecutar una política institucional; o en la inserción en las actividades que ya realiza la institución. Una institución es un organismo público o privado que ha sido fundado para desempeñar labor cultural, científica, política o social, y cumple con una función de interés público.

- Ambientes no institucionalizados. Proyectos que no provienen de los programas de una institución, sino del interés en trabajar alguna problemática en particular a partir de la evaluación, el diagnóstico o la petición de la institución por haber observado alguna situación específica que es de su interés abordar, pero no cabe en las acciones que realizan en la propia institución porque no es su finalidad. Un ejemplo de ello es el trabajo que se puede realizar en una escuela con los alumnos o el profesor, dirigido a la prevención primaria (como prevención de embarazo en la adolescencia), prevención secundaria (como manejo motivacional del alumnado con sobrepeso para iniciar rutinas de actividad física) o prevención terciaria (como intervención en crisis y canalización de un caso de anorexia).

\section{Diferencias entre psicología de LA SALUd Y OTRAS ÁREAS DE APLICACIÓN}

Como se comentó en apartados anteriores, existe una confusión importante que ha dificultado la definición del campo de estudio de la psicología de la salud y las acciones que desde ella se diseñan para intervenir ante asuntos relacionados con los factores psicológicos que inciden en el proceso de salud-enfermedad. Así que a continuación 
se muestran las diferencias entre la Psicología de la Salud y tres áreas con las que con frecuencia se le confunde.

\section{Psicología de la SALUd VS PSicología Clínica}

Piña (2003) afirma que la psicología de la salud y la psicología clínica comparten el objetivo de facilitar y propiciar el desarrollo saludable de la persona consultante; en la práctica están entrelazadas, y para diferenciarlas es necesario discernir el origen y/o motivo de consulta. Se entenderá como una intervención de la psicología de la salud aquella cuyo origen y/o motivo de consulta se encuentre implicado en el proceso de salud-enfermedad (pérdida, disminución, afectación de la salud física) o en la intención de mejorar algún aspecto de la salud física (modificación de hábitos alimentarios, motivación para realizar actividad física, superar la ansiedad vinculada con la revisión médica).

La psicología clínica, en cambio, se encarga de atender los asuntos en que el motivo de la intervención se relaciona con el ajuste psicosocial o la salud mental, como se le llama desde el sistema sanitario, y puede intervenir con individuos, familias o grupos. Su campo de acción ha sido tradicionalmente enfocado en la atención de personas que ya refieren un problema en sus vidas, sus relaciones o una alteración psicológica usando sus propios modelos teóricos para explicar las causas, las consecuencias y las técnicas de intervención de acuerdo con cada modelo. Sin embargo, la intervención clínica también puede usarse en el nivel de prevención primaria y secundaria, sólo que su enfoque se encuentra en el desarrollo de recursos psicológicos para optimizar la capacidad de las personas respecto a su propia vida, la relación familiar y la interacción con otros (Psychology School Guide, 2016).

De modo que si una persona busca ayuda psicológica para superar los efectos emocionales negativos de una separación de pareja, la intervención se enmarca como psicología clínica; si se atiende a una persona con ansiedad que le impide adherirse al tratamiento prescrito por su médico para controlar su diabetes o su obesidad, se está actuando en el campo de la psicología de la salud, aún cuando esa acción sea realizada en el mismo consultorio y por el mismo profesional.

\section{Psicología de la salud vs medicina conduc-} TUAL

El campo de la medicina conductual aborda el trabajo conjunto, integral y complementario hacia la atención de pacientes con problemas crónicos, entre otros, a través de diferentes modelos de intervención, que implican bá- sicamente entrenar a la persona enferma para aprender a vivir en mejores condiciones, evitar deterioro psicológico y conductual para mejorar su calidad de vida.

Florez (2006) plantea que la medicina conductual concentra las aportaciones de diferentes ciencias conductuales y las disciplinas biomédicas para brindar atención integral a la salud, de manera que utiliza diversas técnicas como el biofeedback, registros fisiológicos y otros derivados de la investigación médica aplicada, se apoya en el modelo cognitivo conductual para realizar sus intervenciones. La medicina conductual se desprende de la psicología de la salud pero está enfocada en la prevención terciaria y cuaternaria que fundamentalmente aborda pacientes con enfermedades graves o en fase terminal para lograr adherencia terapéutica, mejor manejo emocional y cognitivo respecto a su enfermedad, el tratamiento y/o el afrontamiento de la muerte, tanto por parte del propio paciente como de su familia (UNAM, 2013).

\section{Psicología de la salud vs psicología comu- NITARIA Y PSICOLOGÍA SOCIAL}

La psicología comunitaria surge en 1965 debido al empeño en desarrollar programas de salud mental dirigidos a la comunidad como beneficiaria, apuntando hacia la prevención primaria y surgiendo como una alternativa a la clínica tradicional donde se concebía que ante una problemática es necesario hacer una intervención, ubicando la relación entre el profesional y las personas como una atención individualizada, en un consultorio. Este modo de ver la salud mental había impedido el desarrollo de estrategias de prevención efectivas y dejaba de lado el entorno de los individuos como agente que influye en los problemas psicológicos, ya que los atribuía sólo a procesos intrapsíquicos.

Los objetivos de esta área son reducir la incidencia y prevalencia de trastornos y alteraciones mentales mediante la prevención y el desarrollo de nuevas formas de tratamiento, así como la promoción del desarrollo sano y la salud mental tanto del individuo como de la comunidad (Gómez del Campo, 2011).

Así que la psicología comunitaria proviene de la psicología clínica, y por tanto no es psicología de la salud a menos que el objetivo de la intervención en una comunidad esté enfocado en los factores psicológicos que afectan la salud física. La comunidad es un contexto en el que la psicología de la salud puede intervenir, pero no es psicología comunitaria.

La psicología social, por otra parte, como una de las grandes ramas de la Psicología, se interesa en el modo en que se influyen mutuamente el individuo y su entorno. 
Así que, usando el conocimiento que aporta la psicología social, se puede buscar comprensión en el proceso de salud-enfermedad en términos del funcionamiento sistémico entre las personas y los fenómenos sociales en lo que respecta, por ejemplo, a la comprensión acerca de la salud, la enfermedad, la influencia cultural en la creencias y prácticas en torno a la salud, por mencionar algunos.

\section{ConCLusiones}

En la descripción que se hace a lo largo de este trabajo queda claro que, aun cuando la psicología de la salud es una disciplina relativamente nueva, ha logrado definir su objeto de estudio, el enfoque de sus intervenciones y los elementos que delimitan su quehacer profesional, así como la intervención que puede realizar en este ámbito, enfocando su actuación en los factores psicológicos involucrados en el proceso de salud-enfermedad.

Sin embargo, el profesional de la psicología que se desempeña en esta área aún tiene retos enormes, ya que la población y las políticas públicas que rigen el sistema de salud no identifican todavía lo que puede aportar para prevenir la enfermedad, promover la salud y mejorar la calidad de vida de las personas y las comunidades. Probablemente esto sea el resultado de la falta de claridad, del propio profesional, respecto al campo específico en que deben circunscribirse sus acciones y el tipo de herramientas que puede usar, que son propiamente psicológicas y que se fundamentan en el conocimiento específico de esta área.

Lo que es importante destacar es que la psicología de la salud es una disciplina destinada a coadyuvar con otras áreas, sin cuyo conocimiento pierde sentido y dirección; sus acciones no sustituyen el papel del médico, del nutriólogo, de la enfermera dedicada a la prevención, sino que aportan elementos teóricos y metodológicos que provienen de la psicología.

De modo que el psicólogo de la salud, por ejemplo, no dará una conferencia acerca del Plato del Buen Comer ni hará sugerencias en torno a la dieta de un paciente, sino más bien realizará acciones dirigidas hacia los factores psicológicos que facilitan el cambio de hábitos alimenticios y se vinculará con otros profesionales para abordar conjuntamente las necesidades específicas detectadas en ese paciente (Piña \& Rivera, 2006).

Así que es posible que la dificultad para definirse como profesional de la psicología de la salud provenga de la falta de claridad acerca de lo que le corresponde aportar, cómo y para qué, aspectos que se vinculan directamente con los retos respecto al actuar del profesional de la psicología de la salud, de los que se hablaba en otro apartado de este documento.
La falta de definición del quehacer de la psicología de la salud comienza en la escasa formación académica y de especialización en esta área, así como de difusión en torno a los resultados de la investigación y el desarrollo teórico metodológico que se ha generado de ella. Ciertamente algunas instituciones educativas en este país han incluido en sus programas materias y formaciones más amplias (áreas de especialización o de posgrado) en psicología de la salud, por lo que se esperaría que en algunos años el profesional de esta área se identifique claramente como tal y sus aportaciones sean, a su vez, identificables por parte de otros. Por lo pronto es necesario que quienes ya se encuentran actuando en este campo logren congruencia entre la práctica y la teoría, que se utilicen y promuevan las herramientas metodológicas que se han desarrollado, que se documenten adecuadamente y se compartan las intervenciones que realizan; en resumen, que se profesionalice y se sustente científicamente el quehacer de la psicología de la salud.

\section{Referencias}

Arco, J. L., \& Fernández, C. A. (Mayo, 2002). Porque los programas de prevención no previenen. International Journal of Clinical and Health Psychology, 2(2), 209-226. Recuperado de http://www.redalyc.org/articulo.oa?id=33720201

Ajzen, I. (September, 2011). The theory of planned behavior: Reactions and reflections. Psychology and Health, 26(9), 1113-1127. Recuperado de https://www.researchgate. net/publication/51653805_The_theory_of_planned. behaviour Reactions and reflections

Bandura, A. (1977). Self-efficacy: toward a unifying theory of behavioral change. Psychological review, 84(2), 191-215.

Bayés, R. \& Ribes, I. E. (1989). Un modelo psicológico de prevención de enfermedad: su aplicación al caso del SIDA. Papeles del psicólogo, 41. Recuperado de http:// www.papelesdelpsicologo.es/resumen?pii=427

Cabrera, A. G. (2000). El modelo transteórico del comportamiento en salud. Revista Facultad Nacional de Salud Pública,18(2),129-138. Recuperado de http://www. redalyc.org/pdf/120/12018210.pdf

Cabrera, A. G., Tascón, G. J., \& Lucumí, C. D. (Enero-Junio, 2001). Creencias en salud: historia, constructos y aportes al modelo. Revista Facultad Nacional de Salud Pública, 19 (1), 91-101. Recuperado de http://www.redalyc.org/ articulo.oa?id=12019107

Enríquez, N. D., Sánchez, M. R., \& Robles, M. S. (2011). Teorías y modelos psicológicos sobre el estudio de la salud sexual. México: UNAM.

Fisher, J. D., \& Fisher, W. A. (2000). Theoretical Approaches to Individual-Level Change in HIV Risk Behavior. In: Peterson J. L., DiClemente R. J. (eds.). Handbook of HIV Prevention. Aids Prevention and Mental Health. Springer, Boston.

Florez, A. L. (Junio, 2006). La psicología de la salud en Colombia. Univ. Psychol. Bogotá, 5(3), 681-693. Recuperado de 
http://www.scielo.org.co/pdf/rups/v5n3/v5n3a20.pdf

García, L. L., Piqueras, J. A., Rivero, R., Ramos, V., \& Oblitas, G. L. (Enero-Junio, 2008). Panorama de la psicología clínica y de la salud. CES Psicología, 1(1), pp. 70-93. Recuperado de http://www.redalyc.org/articulo.oa?id=423539527008

Gervás, J. (2006). Moderación en la actividad médica preventiva y curativa. Cuatro ejemplos de necesidad de prevención cuaternaria en España. Gaceta Sanitaria, 20 (1),127-134.

Gómez del Campo, F. (2011). Psicología de la Comunidad. México: Plaza y Valdés.

Gómez, M. (2007). La psicología de la salud en un hospital de cuarto nivel de complejidad. Psychologia. Avances de la disciplina, 1(2), 159- 179. Recuperado de http://www. redalyc.org/articulo.oa?ld=297224996003

Grau, J. A., \& Hernández, E. (Enero, 2005). Psicología de la salud: Fundamentos y aplicaciones. México: Universidad de Guadalajara.

Juárez, F. (2011). El concepto de salud: Una explicación sobre su unicidad, multiplicidad y los modelos de salud. International Journal of Psychological Research, 4(1), 70-79.

Labiano, M. (2010). Introducción a la psicología de la salud. En L. Oblitas (ed.), Psicología de la salud y Calidad de vida (pp. 3-21). México: Cengage Learning.

Martínez, J. \& García, M. (2012). Epidemiología y salud pública en Promoción de la salud. España: Paraninfo.

Morales, F. (1999). Introducción a la psicología de la salud. Buenos Aires: Paidós Iberica.

Oblitas, L. A. (2008). El estado del arte de la psicología de la salud. Revista de Psicología,26(2), 219-254. Recuperado de http://pepsic.bvsalud.org/pdf/rp/v26n2/v26n2a02.pdf

Piña, L.J. (Marzo, 2003). Psicología clínica y psicología de la salud: En defensa de la psicología de la salud. Suma Psicológica, 10 (1), 67-80.

Piña , L. J. (Diciembre, 2008). Variaciones sobre el modelo psicológico de salud biológica de Ribes: justificación y desarrollo. Universitas Psychologica, 7(1), 19-32. Recuperado de_http://www.scielo.org.co/pdf/rups/v7n1/ v7n1a03.pdf

Piña, L. J., \& Rivera, B. (2006). Psicología de la salud: algunas reflexiones críticas sobre su qué y su para qué. Universitas Psychologica, 5 (3), 669-680.

Piña , L. J., Sánchez, B. C., García, C. I., Ybarra, J. L. \& García, C. H. (Junio, 2013). Psicología y salud en México: Algunas reflexiones basadas en el sentido común y la experiencia. Diversitas: Perspectivas en Psicología, 9(2), 347-360. Recuperado de http://www.redalyc.org/articulo. oa?id $=67932397008$

Prochaska, J. O., DiClemente, C., \& Norcross, J. C. (1994). Cómo cambia la gente. Aplicaciones en los comportamientos adictivos. RET, Revista de Toxicomanías, 1. 3-14.

Psychology School Guide (2016). What is the Difference Between Health Psychology and Clinical Psychology? Recuperado de http://www.psychologyschoolguide. net/guides/difference-between-health-psychology-andclinical-psychology/

Reynoso, L., \& Seligson, I. (2005). Psicología Clínica y de la Salud. México: Manual Moderno.

Robles, S., Solano, R., Díaz, R., Moreno, D., Frías, B., Rodríguez, M., \& Barroso, R. (2012). Efectos de un programa de prevención de problemas de salud sexual sobre variables psicosociales y conductuales en adolescentes sin experiencia sexual. Revista Electrónica de Psicología Iztacala, 15(3), 1129-1155.

Santacreu, J., Marquéz, M., \& Rubio, V. (1997). La prevención en el marco de la Psicología de la Salud. Psicología y Salud. 10, 81-92. Recuperado de https://www.uam.es/personal pdi/psicologia/victor/SALUD/Bibliog/prevencion.PDF

Secretaría de Salud (2015). Norma Oficial Mexicana nom025-ssa2-2014, para la prestación de servicios de salud en unidades de atención integral hospitalaria médicopsiquiátrica. Diario Oficial de la Federación, 4 de septiembre.

Stone, G. C. (1988). Psicología de la Salud: Una definición amplia. Revista latinoamericana de psicología, 20(1), 15-26.

The Association of Faculties of Medicine of Canada (2012). The stages of prevention. Recuperado de http://phprimer. afmc.ca/Part1-TheoryThinkingAboutHealthChapter4Ba sicConceptsInPreventionSurveillanceAndHealthPromoti on/Thestagesofprevention

Universidad Nacional Autónoma de México (2013). Programa de Maestría y Doctorado en Psicología. Medicina Conductual. Recuperado de http://psicologia.posgrado. unam.mx/medicina-conductual-iztacala/

Universidad Nacional Autónoma de México (2012). Coordinación de Universidad abierta y Educación a distancia (SUAyED). Licenciatura en psicología modalidad a distancia. Recuperado de http://suayed.unam.mx/oferta/fichas distancia/lic psicologia distancia.html

Vignolo, J., Vacarezza, M., Álvarez, C., \& Sosa, A. (2011). Niveles de atención, de prevención y atención primaria de la salud. Archivos de Medicina Interna, 33(1), 11-14. Recuperado de http://www.scielo.edu.uy/pdf/ami/v33n1/v33n1a03. pdf 


\section{Meta-Análisis del Artículo}


Revista Digital Internacional de Psicología y Ciencia Social |Vol. 3 | Núm. 2 | Juilio-Diciembre 2017| ISSN 2448-8119 e-ISSN $2448-8119$

\section{Dimensión Cuantitativa}

\section{Perfil de Evaluación entre pares}
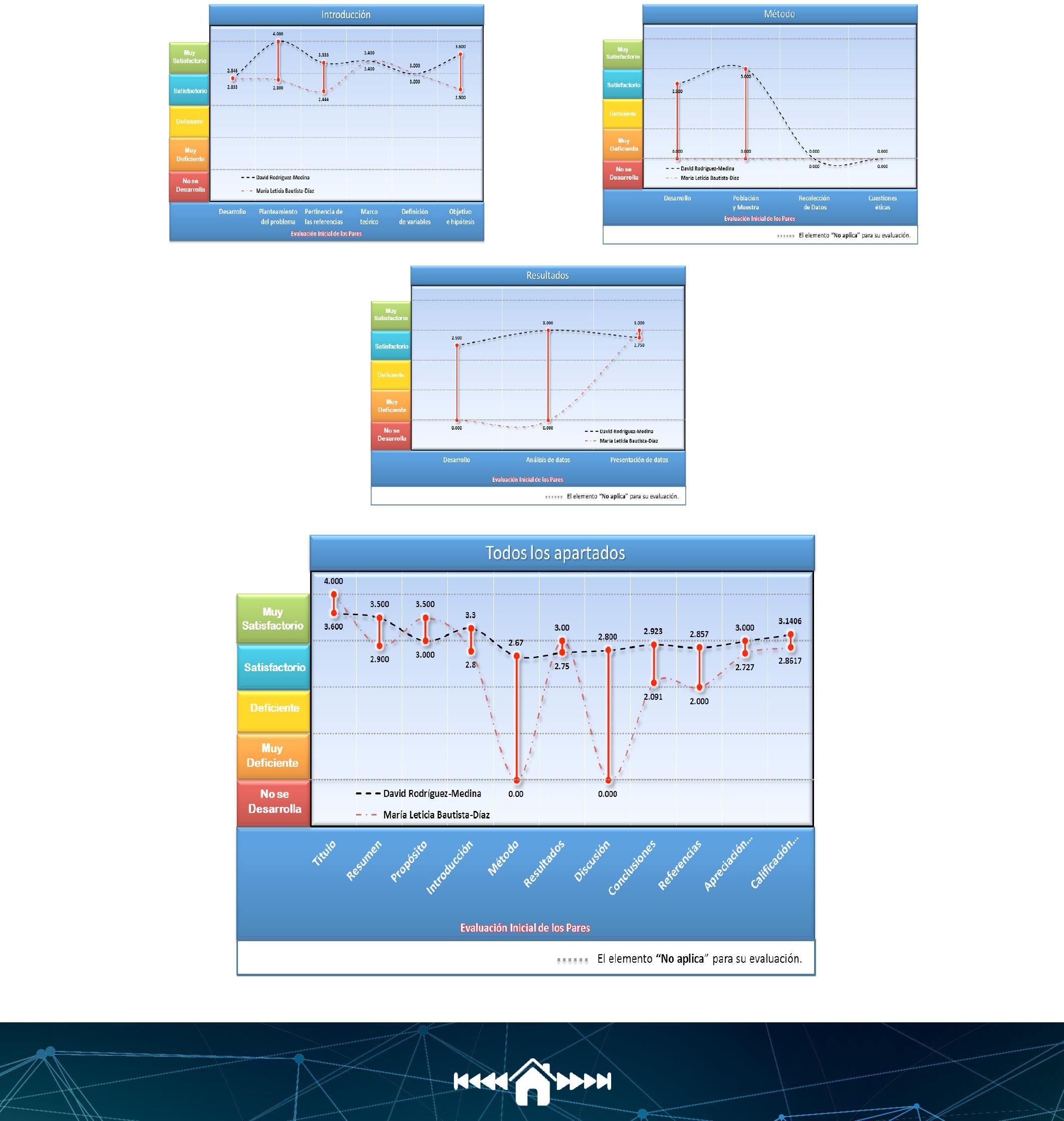


\section{Índice de Concordancia}

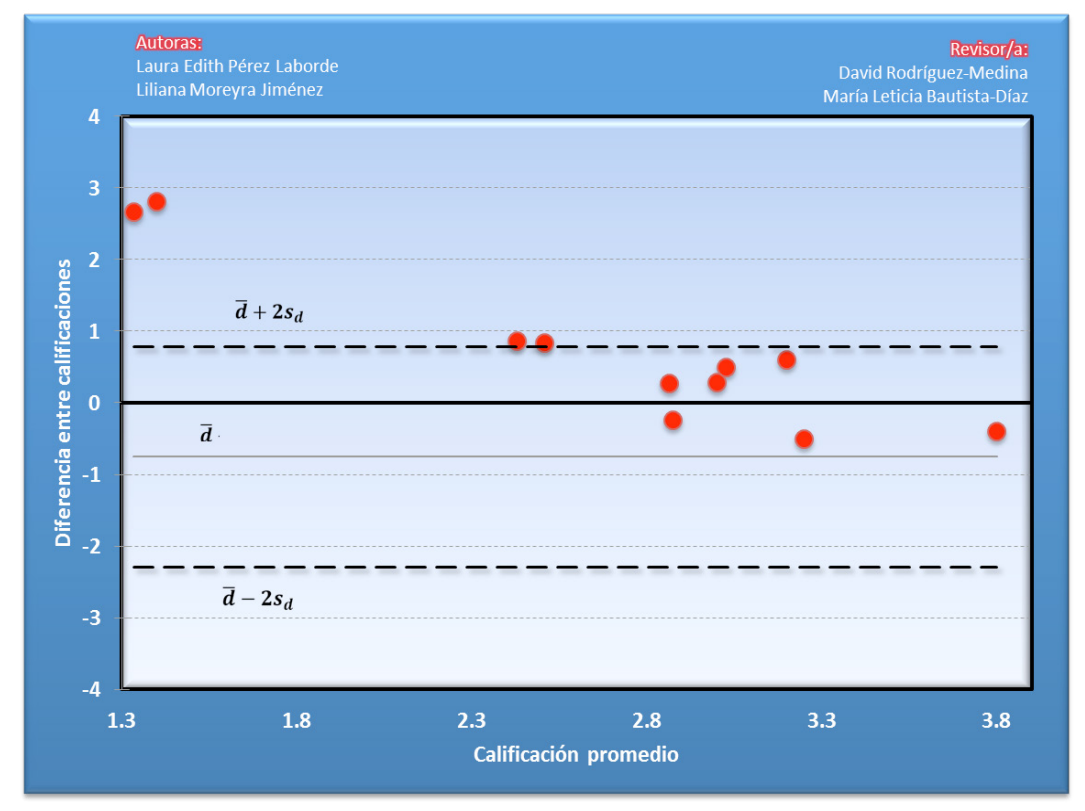

Índice de Acuerdo
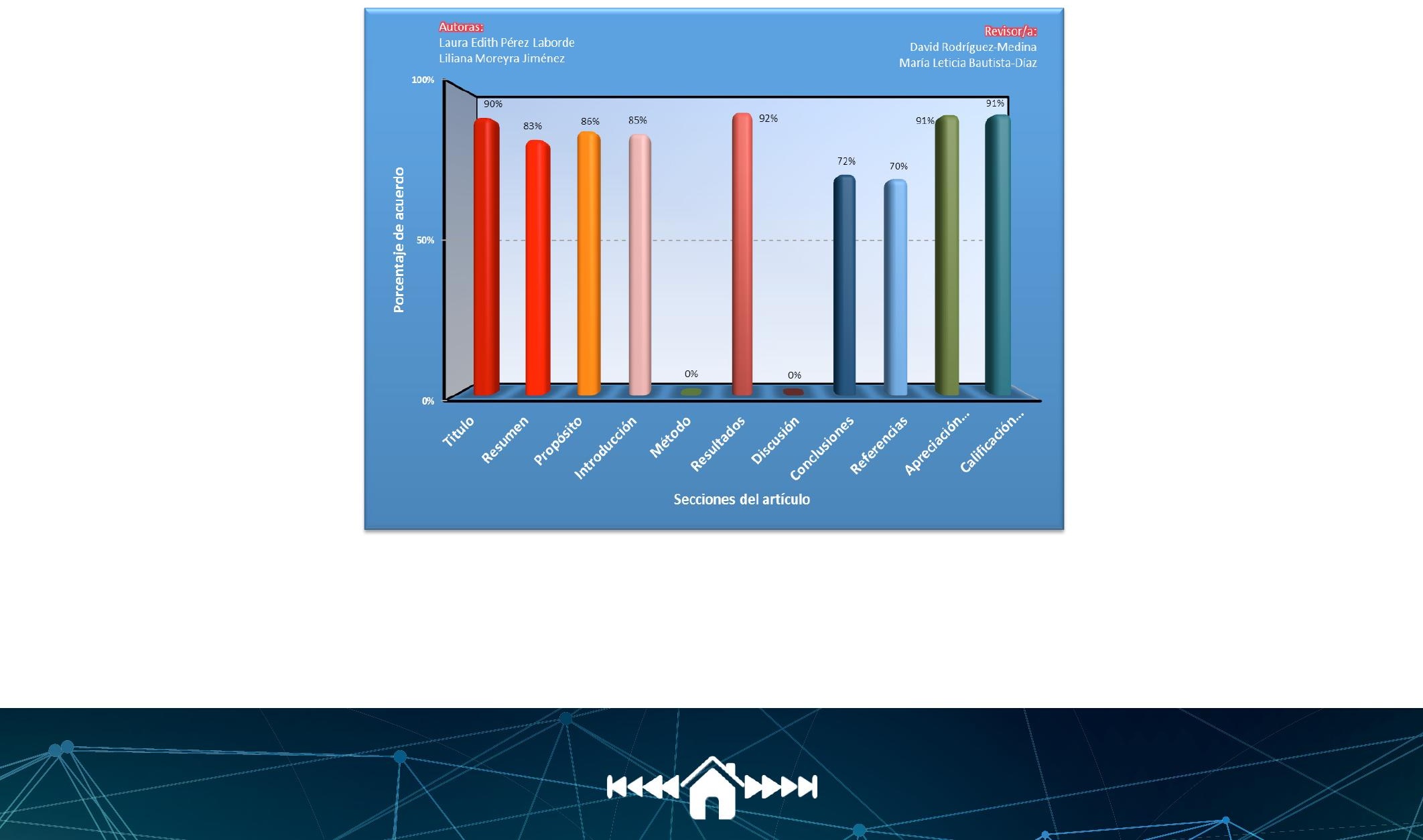
Dimensión Cualitativa

\begin{tabular}{|c|c|}
\hline Revisor 1 & Revisor 2 \\
\hline David Rodríguez-Medina & María Leticia Bautista-Díaz \\
\hline \multicolumn{2}{|c|}{ Título/Autoría } \\
\hline $\begin{array}{l}\text { Sería útil considerar que el título haga alución a los mo- } \\
\text { delos que revisaron, es decir, "EL QUEHACER DE LA } \\
\text { PSICOLOGIA DE LA SALUD: DEFINICIONES, OB- } \\
\text { JETIVOS DESDE LA PERSPECTIVA...” }\end{array}$ & El título es adecuado. \\
\hline \multicolumn{2}{|c|}{ Resumen } \\
\hline $\begin{array}{l}\text { El contenido del resumen es adecuado. Únicamente } \\
\text { deben construirse oraciones más breves, pues el lector } \\
\text { debe hacer un esfuerzo por mantener la información } \\
\text { después de } 3 \text { líneas. }\end{array}$ & $\begin{array}{l}\text { Se refiere que “...en la práctica aún no se encuentra cla- } \\
\text { ramente diferenciada de otras áreas de la psicología, pro- } \\
\text { vocando confusión en quienes buscan intervenir desde } \\
\text { este campo y dificultad en los demás profesionales para } \\
\text { comprender lo que se puede aportar” Lo anterior es un } \\
\text { tanto aventurado decirlo porque no hay sustento que lo } \\
\text { fundamente, quizá las autoras podrían dar un ejemplo de } \\
\text { ello. Se puede enunciar una breve conclusión. }\end{array}$ \\
\hline \multicolumn{2}{|c|}{ Próposito del Estudio } \\
\hline $\begin{array}{l}\text { Revisar y constratar aportaciones internacionales (in- } \\
\text { clyuendo revisiones lationamericanas) con literatura } \\
\text { nacional del tema. }\end{array}$ & Se sugiere "El objetivo del presente trabajo fue..." \\
\hline \multicolumn{2}{|c|}{ Introducción } \\
\hline $\begin{array}{l}\text { Falta constrartar literatura, por ejemplo: el modelo psi- } \\
\text { cosocial con alguno de los modelos biopsicosociales en } \\
\text { materia de salud que clarifique objetivos de la Psicolo- } \\
\text { gía de la salud bajo nuestro contexto Universitario. }\end{array}$ & $\begin{array}{l}\text { Se sugiere incluir otras fuentes para fortalecer las afirma- } \\
\text { ciones ya que, se trata de un de artículo teórico es necesa- } \\
\text { rio ampliar el número de fuentes consultadas, asimismo } \\
\text { se sugiere incluir fuente primarias. En el archivo del ma- } \\
\text { nuscrito, se sugieren diversos cambios o modificaciones, } \\
\text { así como propuestas de redacción. }\end{array}$ \\
\hline \multicolumn{2}{|c|}{ Método } \\
\hline $\begin{array}{l}\text { Sería apropiado mencionar el universo de estudio y su re- } \\
\text { lación temporal con los avances de acuerdo a cada autor/ } \\
\text { modelo revisado. Un diagrama de flujo o bien, una di- } \\
\text { mensión temporal. }\end{array}$ & No aplica. \\
\hline
\end{tabular}




\begin{tabular}{|l|l|}
\hline \multicolumn{1}{|c|}{ Revisor 1 } & \multicolumn{1}{|c|}{ Resultados } \\
\hline $\begin{array}{l}\text { La tabla de niveles de prevención es apropiada de acuerdo } \\
\text { a lo revisado en la literatura. Valdría la pena hacer una si- } \\
\text { milar con los modelos que revisaron sobre los orígenes de } \\
\text { la Psicología de la Salud. }\end{array}$ & No aplica. \\
\hline $\begin{array}{l}|c| \\
\text { Discusión }\end{array}$ \\
\hline $\begin{array}{l}\text { Falta emitir un argumento de las intervenciones en Mé- } \\
\text { xico en Psicología de la Salud en México, que contribu- } \\
\text { ya a los avances de ésta especialidad. }\end{array}$ & No aplica. \\
\hline \multicolumn{2}{|c|}{ Conclusiones } \\
\hline $\begin{array}{l}\text { Faltaría plantear no sólo la utilidad de la Psicología de } \\
\text { la Salud en el contexto nacional, sino una estrategia que } \\
\text { pudiera evaluar sus efectos en sus dimensiones de es- } \\
\text { tudio. Es decir, el lector debe tener claro cómo evaluar } \\
\text { su intervención de acuedo a los intereses del estudio } \\
\text { (clínica, estadística y socialmente). }\end{array}$ & $\begin{array}{l}\text { Se sugiere concluir con la aportación el estudio, ya que se } \\
\text { refiere la falta de claridad del papel del psicólogo de la sa- } \\
\text { lud. La propuesta a partir del estudio es muy valiosa para } \\
\text { el psicólogo de la salud. }\end{array}$ \\
\hline \begin{tabular}{l} 
Sin comenarios. \\
\hline \multicolumn{1}{|c|}{ Referencias } \\
\hline
\end{tabular} & $\begin{array}{l}\text { Se sugiere ampliar el número de referencias, tanto clásicas, } \\
\text { primarias y secundarias para fortalecer las afirmaciones. } \\
\text { No aparecen los autores de los modelos de salud (Hack- } \\
\text { baum (1950), Prochaska (1970) y Fishben. Se sugiere in- } \\
\text { cluirlas. En el archivo del manuscrito se indican una serie } \\
\text { de detalles por corregir en las referencias. }\end{array}$ \\
\hline
\end{tabular}


